\title{
Inevitable variance of electric field of plasma membrane
}

\author{
Questioning the foundation of current physiology
}

\author{
Bernard Delalande \\ Hirohisa Tamagawa \\ Titus Mulembo \\ Vladimir Matveev
}

\begin{abstract}
An embryonic version of membrane theory can be date back to the Bernstein's work reported more than a hundred years ago. Such an originally old work has evolved conceptually and mathematically up until today, and it plays a central role in current membrane theory. Goldman-Hodgkin-Katz equation (GHK eq.) is one of the math-based monumental works, which constitutes the present membrane theory. Goldman theoretically derived GHK eq., but its physiological meaning was provided by the two renowned scientists, Hodgkin and Katz. These two employed an assumption that the electric field (EF) across the plasma membrane is constant to validate the GHK eq. physiologically. Proposal of Hodgkin-Huxley model ( $\mathrm{HH}$ model) is another math-based monumental works developed from the membrane theory and now forms a fundamental part of the current membrane theory. GHK eq. and HH model are quite fundamental central concepts in the current physiology. Despite the broad acceptance of GHK eq. at present time, its prerequisite that the EF within the plasma membrane is constant is hardly believable. Especially when the action potential is generated, it sounds totally nonsense. Furthermore, the
\end{abstract}

Bernard Delalande

280, avenue de la Pierre Dourdant, 38290 La Verpilliere, France

Tel.: +33-6 07274753

E-mail: bernard@somasimple.com

Hirohisa Tamagawa, Titus Mulembo

Department of Mechanical Engineering, Faculty of Engineering, Gifu University, 1-1 Yanagido, Gifu, Gifu, 501-1193 Japan

Tel.: +81-58-293-2529

Fax: +81-58-293-2529

E-mail: tmgwhrhs@gifu-u.ac.jp, titusmulembo@gmail.com

Vladimir Matveev

Laboratory of Cell Physiology, Institute of Cytology, Russian Academy of Sciences, Tikhoretsky Ave 4, St. Petersburg 194064, Russia

Tel: +7931951 5112

E-mail: vladimir.matveev@gmail.com 
existence of constant $\mathrm{EF}$ within the plasma membrane is conceptually almost in conflict with the $\mathrm{HH}$ model. The authors will discuss those problematic issues the membrane theory inherits.

Keywords membrane potential · constant electric field · resting potential · action potential · GHK eq. · HH model

\section{Introduction}

Bernstein built the earliest foundation of current membrane theory. He proposed that a semi-permeable membrane surrounds a living cell. He even quantitatively measured action potential and pointed out the occurrence of permeability change of cell membrane to mobile ions. His theory has evolved into the current membrane theory now broadly admired. The membrane theory assumes that the membrane is under the influence of an electric field (EF) 1, 2]. This statement comes from the simple fact that it is possible to measure a potential difference between the external milieu and the cell-interior. This potential difference is negative by definition, the cell-interior being more negative than the cell-exterior. The potential assumes either two states: a stable almost constant state called resting potential or an excited state which involves the generation of commonly called action potential. The cell switches from the resting state to the excited state by depolarization.

The notion of a constant EF within a plasma membrane is one of the fundamental bases of the GHK eq. representing membrane potential, a typical expression is given by Eq.1 Without the constant EF condition of the plasma membrane, Eq. 1 cannot be derived. However, the authors think that this condition "EF within the plasma membrane is constant," is often dismissed.

$$
\phi=-\frac{k T}{e} \ln \frac{P_{K}\left[K^{+}\right]_{i n}+P_{N a}\left[N a^{+}\right]_{i n}+P_{C l}\left[C l^{-}\right]_{o u t}}{P_{K}\left[K^{+}\right]_{o u t}+P_{N a}\left[N a^{+}\right]_{o u t}+P_{C l}\left[C l^{-}\right]_{i n}}
$$

Although Hodgkin and Katz gave contemporary physiological significance to GHK eq. by considering independent diffusion of ionic species and even electrical diffusion through the membrane [3], some aspects of electrochemistry do not seem to allow such simplification or approach. For example, Goldman stated to the effect that the assumption - the EF is constant in the transversal direction of the plasma membrane - is inappropriate.

The HH model is a monumental accomplishment of electrophysiological science as well as GHK eq. But is it built on a perfectly sound scientific foundation? Is it still a model to be preserved and taught, despite the fact that it contains contradictions that some scientific disciplines cannot tolerate? Biology and biophysics must respect the constraints and limits imposed by the laws chemists and physicists have found. However, $\mathrm{HH}$ model does not seem to be in line with, for example, GHK eq.

In physiology, it is acknowledged that the action potential (AP) generation is due to membrane permeability change to individual mobile ions. Such an 
effect is incorporated into the GHK eq. as a change of permeability, $P$. But the change of $P$ means the change of plasma membrane status, and it strongly suggests that the local variation of EF inevitably takes place. It conflicts with the constant EF generation assumption regardless of the location within the plasma membrane. One may say that EF could be a time-dependent quantity but it could be constant within the whole body of plasma membrane. However, it is intuitively quite unlikely to take place. Hence, there must be something incomplete in the current physiological foundation. Still someone may argue that the GHK eq. is valid only for the analysis of resting potential and $\mathrm{HH}$ model should be employed for the analysis of AP characteristics. Yes, that's one of what we would like to say, the GHK eq. cannot be used for the analysis of AP. AP generation is inevitably accompanied by the local variation of EF, which conflicts with a requirement for validating GHK eq.

In this work, the authors would like to discuss the problematic facets of current membrane theory, especially in light of EF within the plasma membrane.

\section{Questionable notions about EF in a plasma membrane}

\subsection{Extraordinarily high EF}

AP must be caused by a transient and spatially local variation in the membrane condition. AP takes place at only a small portion of the axon, unlike an electric cable where the entire conductor sees a voltage change according to the applied signal. The well-known $\mathrm{HH}$ model explains this variation in membrane potential primarily by two ionic flows [4,5,6]: one composed of sodium ion flow across a plasma membrane, and the other is potassium flow across a plasma membrane in the opposite direction. Therefore, the theory proposes the flow composed of ions, all positive, in perfectly opposite directions for most of AP's duration. Does the presence of a constant EF confirm this hypothesis? or Doesn't it impose limitations on such ionic currents? To better understand the problems raised about the theoretical model during the AP's induction, it is desirable to reflect on $\mathrm{EF}$ and the ions under the EF.

EF flux lines orient from the positively charged zone to the negatively charged zone [7. This implies that the EF can influence the movement of charged particles. A neuron's membrane thickness is estimated to be between 4 and $5 \mathrm{~nm}$. The resting potential of the membrane is around $-75 \mathrm{mV}$. These values can allow us to compute $\mathrm{EF}, E$, which is proportional to the potential difference of $V$, as long as EF is constant.

$$
\begin{gathered}
E F=\frac{V}{d} \approx 1.5 \times 10^{7} V^{-1} \\
E F \propto V
\end{gathered}
$$

This value is already in itself, extraordinary and contradictory. $|E F|$ is not only nonzero but extraordinarily high for a plasma membrane. Indeed, 
Goldman points out in his work that it is impossible to approach such values with samples exposed even to a much lower EF, say a few thousand volts per centimeter. Even some samples, reputed to be robust, were permanently damaged [2]. Even the plasma membrane, of a thinner and less homogeneous thickness, cannot then in any way be a better dielectric. In addition to that, such a high EF exerts unusually high force to individual ions. There are some reports that $\mathrm{Cl}^{-}$does not influence on the membrane potential generation [3]. Why isn't the $C l^{-}$taken into consideration? Doesn't such a high EF have any influence on the flow of $\mathrm{Cl}^{-}$bearing negative charge? What distinguish $\mathrm{Cl}^{-}$ from sodium and potassium? It is, therefore, questionable if such a high EF is relevant.

\subsection{Geometry of plasma membrane}

The cross-sectional view of plasma membrane is like a curved wall as illustrated in Fig. 1. Of course, the geometry of real living cell is not such simple. But for simplifying the discussion about the EF within the plasma membrane, the authors assume that the cell takes the simple structure just like this illustration. Membrane theory states that EF from A to B (see Fig. 1) within the plasma membrane is nonzero and constant. In addition to it, the membrane theory even suggests that the EF within the plasma membrane never varies from place to place within the plasma membrane. It is geometrically hardly believable as explained next.

The dotted line circumference of drawn in the plasma membrane is shorter than that of dashed line (see Fig. 1). Although EF depends on the charge distribution in the cell, it is quite unlikely that the charges distribute so that the $\mathrm{EF}$ along the line $\mathrm{AB}$ is nonzero constant. Please let the authors discuss it more by employing a simpler system. According to the electromagnetism, the electric field generated by a point charge, Q, in the space is theoretically predictable using Gauss's theorem. Eq. 4 gives the electric field, E, generated at distance R away from the point charge Q. Gauss's theorem indicates that the EF within the plasma membrane almost inevitably changes in accordance with the distance from the charge spatially fixed.

$$
\oint_{S} d S \cdot E=\frac{Q}{\epsilon_{0}}
$$

\subsection{Ion transport by diffusion}

Diffusion is a major force of ion transport in a living cell, and it has almost become an undeniable dogma. Fick's work has focused on the diffusion of electrically neutral saline solutions. Biologists have extended this rule, without precaution, to the diffusion of electrically charged solutions. Nernst's discoveries require that the ionic movement between two compartments requires 


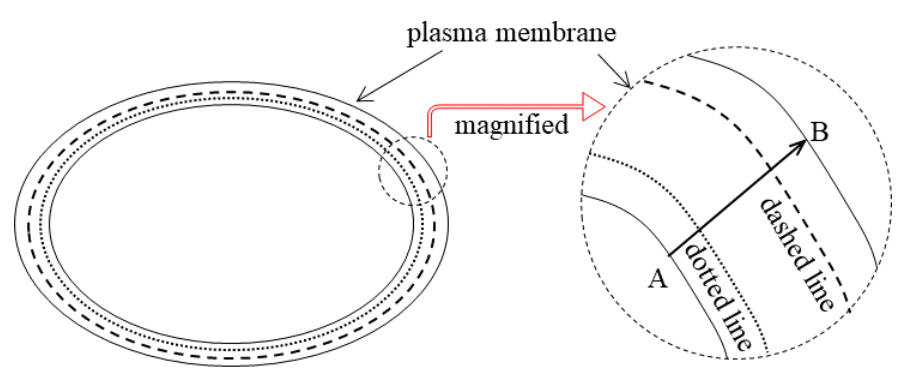

Cell

Fig. 1 Plasma membrane structure and EF

chemical reactions and electron transfer. This mandatory condition is not met in the ion diffusion described by biologists.

A diffusion alone is unthinkable about the electrolytic solution system without chemical energy supply. [?]. Petrucci says "Chemical reactions involve the making and breaking of chemical bonds (ionic and covalent) and the chemical energy of a system is the energy released or absorbed due to the making and breaking of these bonds. Breaking bonds requires energy, forming bonds releases energy, and the overall reaction can be either endergonic $(\triangle G<0)$ or exergonic $(\triangle G>0)$ based on the overall changes in stability from reactants to products" [8. Therefore, it is quite natural to think that the enormous quantity of energy must be involved in the ion transport in the living cell system. However, Ling has already elucidated that a single living cell does not have enough energy to transport the mobile ions through the plasma membrane as believed [?].

\section{Revisiting the E-field within a plasma membrane}

$\mathrm{K}^{+}$concentration in the cell-interior is greater than that in the cell-exterior in the resting state, while $\mathrm{Na}^{+}$concentration in the cell-exterior is greater than that in the cell-interior. Due to the ion concentration disparity, ions tend to diffuse to either cell-exterior or cell-exterior across the plasma membrane. It is quite natural that such in diffusion eventually nullify the ion concentration disparity. However, actual living cell never loses such ion disparity. According to the commonly accepted physiological concept, the disparity of ion concentration is achieved by the functionality of ion channels and sodium pumps embedded in the plasma membrane. Ion transport at the resting state is achieved by the equilibrium between the passive ion transport and active ion transport. EF within the plasma membrane in the resting state is extremely high as described earlier. Hence, sodium is transported from cell-interior to cell-exterior against such a high EF in a plasma membrane. However, Ling suggests that a living cell is not possessed of enough energy for operating 


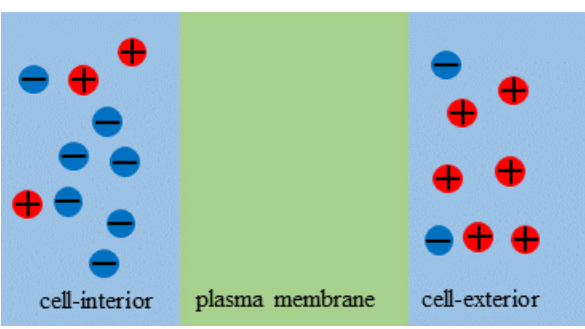

Fig. 2 Homogeneous ion distribution (Mobiles ions only are drawn.)

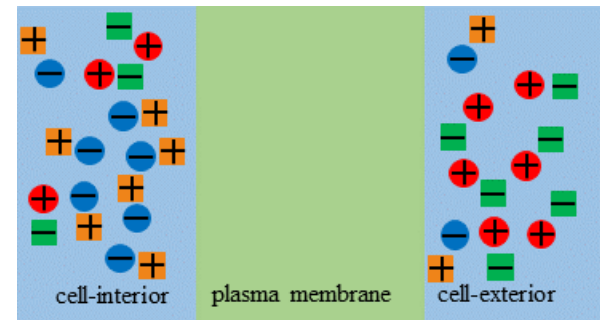

Fig. 3 Homogeneous ion distribution where all the mobile ions are neutralized by neighboring immobile ions.

$\mathrm{Na}^{+} / \mathrm{K}^{+}$pump. In addition to it, a quite high EF exists within the plasma membrane. So, the occurrence of ion transport against such a high EF and against such a high ion concentration gradient is not plausible thermodynamically. Therefore, such a problematic point should be addressed. The living cell system is viewed as a three-phase system in this section, cell-interior, plasma membrane and cell-exterior.

How is such a high EF generated in the plasma membrane? Considering the experimental fact that the cell-interior potential is lower than that of cell-exterior in the resting state, it is quite natural to imagine that the cellinterior contains a greater quantity of anions than the cell-exterior does, while the cell-exterior contains a greater quantity of cations than the cell-interior does. However, it is hardly imaginable that the greater quantity of anions homogeneously distributes in the cell-interior, while the greater quantity of cations homogeneously distributes in the cell-exterior as illustrated in Fig. 2 Ion distribution should obey the macroscopic electroneutrality. Therefore, any charged particle has to be accompanied by the particles bearing the charge of opposite sign so as to be electrically neutralized. Hence, the system shown in Fig. 2 should contain the immobile ions which can fully neutralize the mobile ions as illustrated in Fig. 3. So, the nonzero potential generation across the plasma membrane cannot be expected. Nonzero potential generation is explicable only by the heterogeneously but locally distributing charges along with sufficing the macroscopic electroneutrality. 


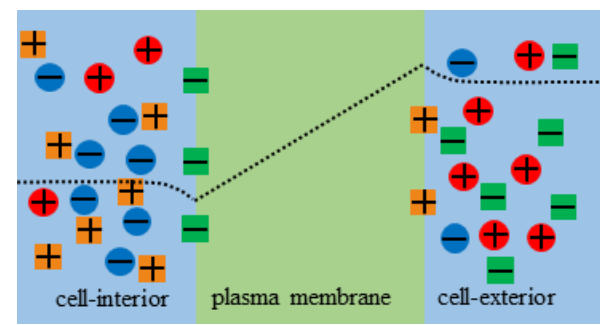

Fig. 4 Local heterogeneous ion distribution Dotted line represents the expected potential profile.

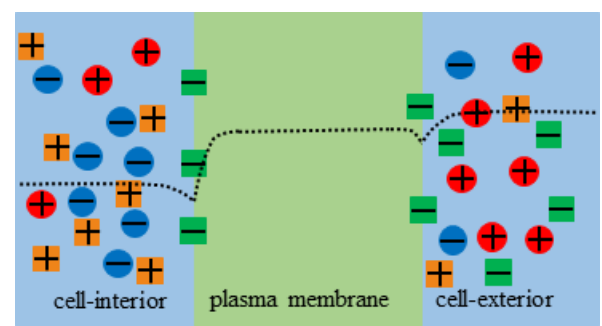

Fig. 5 The expected potential profile when the negative charges distribute at the interface between two phases.

We think that the plausible condition is depicted in Fig. 4 , it represents the emergence of microscopic local nonzero charge distribution. Spatially fixed anions are at the interface between the cell-interior and the plasma membrane, while the spatially fixed cations are at the interface between the cell-exterior and the plasma membrane. Spatial localization of such a small quantity of ions is allowable even from the view of thermodynamics. But still such a situation shown in Fig. 4 is unlikely to be realized by the following reason: Plasma membrane surface is primarily covered with negative charges of lipids. Hence, the more plausible ion distribution and potential profile is given by Fig. 5 But the potential profile within the plasma membrane shown in Fig, 5 is in conflict with the basic assumption of GHK eq. that the EF within the plasma membrane is constant. As clearly seen in Fig. 5. the EF is not constant at the interface of neighboring phases, but thermodynamically allowable. But should the EF within the plasma membrane be constant? or Can the EF be uniformly constant everywhere in the plasma membrane?

Now, let's consider more about the ion transport with the active state in mind along with the resting state. The charge distribution at the resting state is represented by Fig. 2 according to the common physiological concept. Namely, the cell-interior contains the excess quantity of negative change, while the cell-exterior contains the excess quantity of positive charge, and those charges distribute homogeneously in the individual phases. In the resting state, $\mathrm{K}^{+}$ concentration in the cell-interior is greater than that in the cell-exterior, while 
$\mathrm{Na}^{+}$concentration in the cell-exterior is greater than that in the cell-interior. Since the excess quantity of negative charge in the cell-interior and the high EF directed toward the cell-interior, $\mathrm{K}^{+}$must have a great tendency to stay in the cell-interior, and in fact, the concentration of $\mathrm{K}^{+}$in the cell-interior is greater than that in the cell-exterior. It is expected that $\mathrm{Na}^{+}$has a great tendency to stay in the cell-interior, too, since $\mathrm{Na}^{+}$is positively charged ion just like $\mathrm{K}^{+}$. However, the actual concentration of $\mathrm{Na}^{+}$in the cell-interior is by far less than that in the cell-exterior. The cell-exterior contains the homogeneously distributing positive ions. Hence, $\mathrm{Na}^{+}$must be under the great repulsive force from the cell-exterior. Nevertheless, the greater quantity of $\mathrm{Na}^{+}$stay in the cell-exterior. It has been broadly accepted that such ion distribution disparity is achieved by the pump's functionality, and it forcibly transports $\mathrm{Na}^{+}$from the cell-interior to the cell-exterior. Factors which cause the ion concentration disparity are summarized in Tables 1 and 2 . So, for both $\mathrm{K}^{+}$and $\mathrm{Na}^{+}$, five factors - excess quantity of anion in the cell-interior, the extraordinarily high $\mathrm{EF}$ in the plasma membrane, sodium pump in the plasma membrane, an excess quantity of cation in the cell-exterior, the gradient of ion concentration between the cell-interior and the cell exterior - are dominant factors for the living cell in the resting state of a living cell. As to $\mathrm{K}^{+}$ion concentration disparity, four factors except for gradient of $\mathrm{K}^{+}$concentration play a role for maintaining the $\mathrm{K}^{+}$concentration disparity between the cell-interior and the cell-exterior. On the other hand, four factors except for sodium pump play a role as the counter-factors for maintaining the $\mathrm{Na}^{+}$concentration disparity between the cell-interior and the cell-exterior. Hence, pump takes huge energy for achieving the $\mathrm{Na}^{+}$concentration disparity.

Table 1 Factors and counter-factors for achieving the concentration disparity of $\mathrm{K}^{+}$

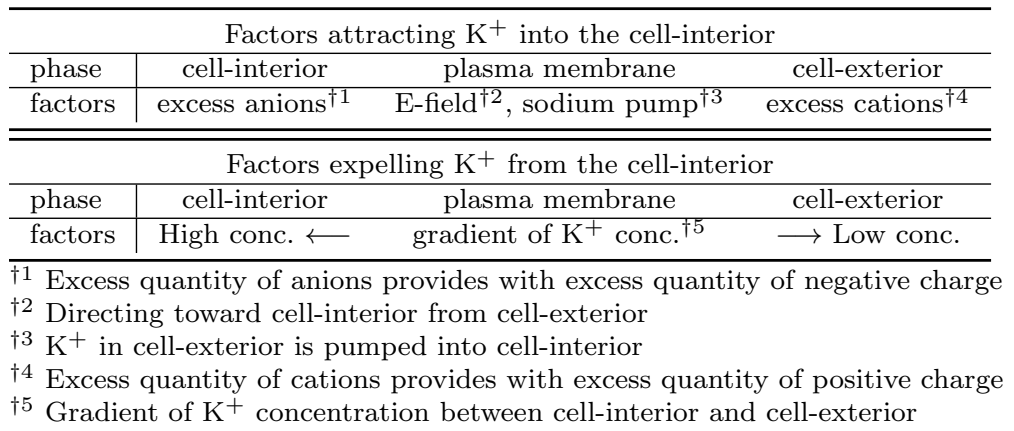

At the onset of the transition of membrane potential from the resting to the active takes place, membrane potential soars from negative to positive. Current physiological concept states that this potential soaring is caused by the influx of $\mathrm{Na}^{+}$from the cell-exterior to the cell-interior. This soaring of potential is followed by the fast plunge of potential by the efflux of $\mathrm{K}^{+}$from 
water is low. Hence, the ions hardly enter the cell-interior even though the plasma membrane does not exist. But Ling observed that $\mathrm{K}^{+}$entered the cellinterior and stayed in the cell inside, why ? Because of the thermal motion and smallness of hydrated $\mathrm{K}^{+}$, some hydrated $\mathrm{K}^{+}$'s entered the cell inside and came to be adsorbed on to the adsorption sites of the protein. Thus, some $\mathrm{K}^{+}$'s were accumulated in the cell inside without channels and pumps. The authors wonder how the current physiological theory explains the EMOC experimental result. What plays the role of transporter for mobile ions. Without sodium pumps, $\mathrm{Na}^{+}$cannot be expelled from the cell-interior as easily understood by considering the ion transport factors summarized in Table 2. Ling thinks that there is something wrong with the membrane theory.

\section{Consequences for the HH model.}

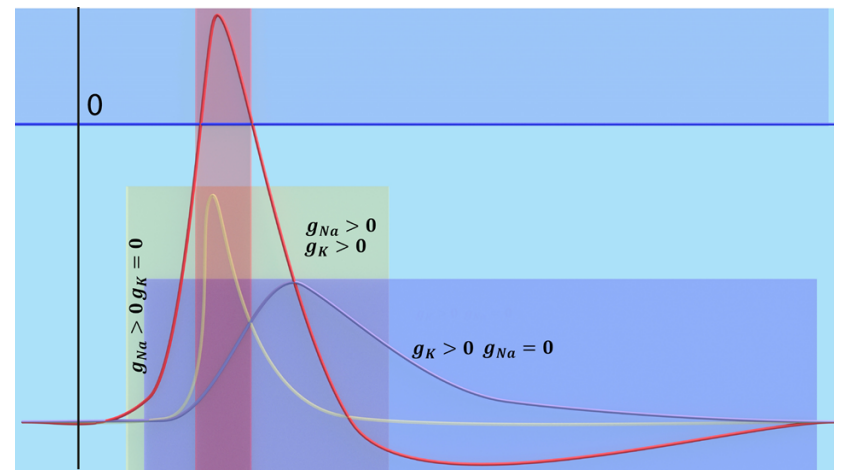

Fig. 6 The action potential as recorded by Hodgkin and Huxley (in red), the conductance calculated in the HH model of sodium (in yellow) and potassium (in purple). The pale red area represents the area where the EF changes direction. The reference voltage $(0.00 \mathrm{~V})$ is represented by a blue line. The different values of the conductance are indicated on the horizontal time axis.

Here we would like to discuss the electrical characteristics of a living cell in active state in detail. The typical action potential profile is shown in Fig. 6. HH model is a broadly used tool for quantitatively assessing the action potential. Conductance is a characteristic distinguishing the $\mathrm{HH}$ model. The behavior of conductance is given in Fig. 6 along with the action potential profile. There has been no questioning that the diffusion phenomena would be the origin of most of the resting potential and the action potential, and an orchestrated variation in sodium and potassium conductance is a cause of the action potential induction. 
4.1 Paradox of potassium conductance

The HH model was proposed to solve the voltage variations of the action potential by using mathematical equations. It seems that the solution adopted resembles a curve fitting satisfying an expectation. This is like simulating incomplete data. Of course, this does not diminish themathematical demonstration's elegance, but it does not always guarantee the model's accuracy for explaining nature. It is recognized that resting potential depends on high potassium conductance. This is what the GHK equation highlights by predicting the predominance of permeability $P_{K}$ compared with the membrane permeability to other mobile ions. However, the conductance curves established according to the $\mathrm{HH}$ model show the conductance of potassium is equal to that of sodium, what is more, they are almost zero (Eq. 5. This is a paradox that is difficult to solve. One may argue that GHK eq. cannot deal with the action potential, though $\mathrm{HH}$ can, hence $\mathrm{P}_{K}$ of $\mathrm{GHK}$ eq. and $g_{K}$ of $\mathrm{HH}$ mode should not be discussed on a equal footing each other. However, the authors are discussing the resting state and the resting potential at this moment.

$$
g_{K}=g_{N a} \approx 0
$$

Bernstein's potassium hypothesis tells us that the cell-interior in the resting state contains more potassium than the cell-exterior and that diffusion should require an outflow. The GHK equation, in the same way, states that potassium is the main source of membrane potential and indicates that permeability to the membrane has a predominant quantity. Since permeability is proportional to conductance, it must be concluded that potassium conductance is higher than sodium and higher than 0 . But the $\mathrm{HH}$ model never gives us such an outcome.

\subsection{Independence of ion mobility}

Assuming that there the high EF exists within the plasma membrane, the $\mathrm{HH}$ model and the GHK equation ignore the electrostatic forces generated by the EF. They do not take into consideration the physical location of mobile ions. If we grant an independent movement of ions that violates the macroscopic electroneutrality, the ion movement under nonzero EF must result in a model that cannot reflect the experimental facts.

It is suggested in the theory that the sodium flow enters the cell-interior and the potassium flow is outgoing in the active state. But the extraordinarily high EF never allow this. It's not an option. Of course, the EF in the plasma membrane is not always extraordinarily high in the active state. However, the status of plasma membrane bearing the extraordinarily high EF inevitably takes place at a certain point in the AP generation process. Then, how does the living cell transport $\mathrm{Na}^{+}$against such a high EF. 


\section{Plasma membrane without high EF}

One of the authors of this paper, H.T., and his collaborators suggested that an identical equation to the GHK eq. can be derived without assuming the constant EF of plasma membrane. H.T. et. al found that attributing the membrane potential generation to the ion adsorption process rather than to the ion transport across the plasma membrane, such an equation is derived. In addition to that, the generation of high EF does not show up as long as the membrane potential generation mechanism is attributed to the ion adsorption-desorption process. Further investigation by H.T. and his other collaborators even found that GHK eq. and HH model are mathematically associable with each other as long as the membrane potential generation mechanism is attributed to the ion adsorption-desorption process.

\section{Conclusions}

Membrane theory a number of renowned scientists built has significantly promoted research in biology and biophysics. Their work is, however, as usual, an attempt to explain the phenomena observed. They even expressly stated that their vision of things was incomplete and could not cover all aspects of the complexity of the facts. They specified the limits and conditions of application through experiments and rules. Hence, biology needs to use other sciences that have described as aspects of physics. It is surprising that some sciences, particularly the life sciences, do not call upon specialists in fields where they may encounter difficulties when necessary.

The models in life science deserve to be reviewed with multidisciplinary teams that encourage exchanges and open-mindedness and, above all, the rewriting of theories with stronger scientific foundations and better logic. To say nothing of the membrane theory, the well-known $\mathrm{HH}$ model, for instance, presents contradictions regarding the initial conditions such that the potassium conductance value is not in conformity for the resting potential. The presence of an EF disrupts or even cancels all ionic exchanges as described. The model, therefore, cannot describe how the neuron works.

\section{References}

1. J. Bernstein, A. Tschermak, Untersuchungen zur Thermodynamik der bioelektrischen Ströme: Zweiter Teil Über die Natur der Kette des elektrischen Organs bei Torpedo, Pflüger, Archiv für die Gesammte Physiologie des Menschen und der Thiere 112(910), 439 (1906). DOI 10.1007/BF01676972. URL http://link.springer.com/10.1007/ BF01676972

2. D.E. Goldman, POTENTIAL, IMPEDANCE, AND RECTIFICATION IN MEMBRANES, The Journal of General Physiology 27(1), 37 (1943). DOI 10.1085/jgp.27.1.37. URL http://www.jgp.org/cgi/doi/10.1085/jgp.27.1.37

3. G.N. Ling, A revolution in the physiology of the living cell, original ed. 1992 edn. (Krieger Pub. Co, Malabar, Fla, 1992) 
4. A.L. Hodgkin, P. Horowicz, The influence of potassium and chloride ions on the membrane potential of single muscle fibres, The Journal of Physiology 148, 127 (1959). DOI 10.1113/jphysiol.1959.sp006278

5. A.L. Hodgkin, A.F. Huxley, A quantitative description of membrane current and its application to conduction and excitation in nerve, The Journal of Physiology 117(4), 500 (1952). DOI 10.1113/jphysiol.1952.sp004764. URL https://onlinelibrary.wiley . com/doi/abs/10.1113/jphysiol.1952.sp004764

6. A.F. Huxley, R. Stampfli, Effect of potassium and sodium on resting and action potentials of single myelinated nerve fibers, The Journal of Physiology 112(3-4), 496 (1951). DOI 10.1113/jphysiol.1951.sp004546

7. O.D. Jefimenko, Electricity and magnetism: an introduction to the theory of electric and magnetic fields, 2nd edn. (Electret Scientific, Star City, 1989)

8. R.H. Petrucci, General chemistry: principles and modern applications (Pearson Canada, Toronto, 2017) 